\title{
Tranexamic Acid
}

National Cancer Institute

\section{Source}

National Cancer Institute. Tranexamic Acid. NCI Thesaurus. Code C47765.

A synthetic derivative of the amino acid lysine with antifibrinolytic activity. With strong affinity for the five lysine-binding sites of plasminogen, tranexamic acid competitively inhibits the activation of plasminogen to plasmin, resulting in inhibition of fibrinolysis; at higher concentrations, this agent noncompetitively inhibits plasmin. This agent has a longer half-life, is approximately ten times more potent, and is less toxic than aminocaproic acid, which possesses similar mechanisms of action. 